\title{
A simple method for displaying Fresnel holograms on liquid crystal panels
}

\author{
R. Tudela*, I. Labastida, E. Martín-Badosa, S. Vallmitjana, I. Juvells, A. Carnicer \\ Departament de Fúsica Aplicada i Òptica, Universitat de Barcelona, Diagonal 647, E 08028 Barcelona, Spain
}

\begin{abstract}
In this paper we present a method for reconstructing Fresnel holograms using two liquid crystal devices, one to display the amplitude information and the other to display the phase. The theoretical approach has been adapted to real configurations of VGA panels removed from a commercial video projector. The optical setup is based on the projection of the phase plane into the amplitude plane by means of an imaging lens. Simulated and experimental results are presented.
\end{abstract}

Keywords: Fresnel holography; Wave front reconstruction; Liquid crystal devices; Full-complex modulation

\section{Introduction}

Since the generalized access to liquid crystal displays (LCD), these devices have become the most frequently used spatial light modulators (SLM) in the field of optical image processing. The modulation properties of LCDs allow a real time manipulation of a wave front, therefore it is possible to display holographic information using them. This operation requires the display of complex functions in order to modify the amplitude and phase of a wave. Most of the present LCDs provide a wide range of operating modes but none

\footnotetext{
${ }^{*}$ Corresponding author. Fax: +34-93-402-1142.

E-mail address: raul@optica.fao.ub.es (R. Tudela).
}

of them allows the complete display of a fullcomplex plane.

There are different strategies to achieve fullcomplex modulation. A first approach consists in codifying the complex information to be displayed on a single device with limited modulation capabilities. Several methods of codification that transform the complex information into real positive values to be displayed on a transmissive element are reported in [1]. Recently new approaches were proposed in [2,3]. Birch et al. [2] use an analog ferroelectric reflective SLM where the information is codified using a macro-pixel composed by two pixels of consecutive rows, the first pixel containing the real data and the second one containing the imaginary data. All these methods that make use of several pixels to codify the information entail a loss of resolution. On the 
other hand, Stolz et al. [3] have studied the properties of the coupled phase and amplitude domains in the implementation of diffractive optical elements (DOEs) onto a single SLM, compared to the use of amplitude-mostly or phase-mostly modes.

A second approach to obtain full-complex modulation is to couple two SLMs. In this case two issues have to be considered, the coupling optical setup and the modulation capabilities of each device. Juday and Florence [4] and Gregory et al. [5] proposed the two common optical systems to combine two devices: an architecture for combining amplitude and phase as a sum and another one for combining both parts as a product (cascade configuration). Amako et al. [6] and Gonçalves et al. [7] use a $4 f$-cascade architecture to obtain full-complex modulation taking into account the operating configurations of the coupled SLMs. Amako et al. use a twisted-nematic liquid crystal (TNLC) device for amplitude modulation, and an electrically controlled birefringent (ECB) device for phase modulation and display both data separately. Instead, Gonçalves et al. use two TNLC SLMs in amplitude and phase regimes but combine the two coupled modulations into the required complex modulation by applying a lookup table encoding method.

In this paper we present a method to reconstruct Fresnel holograms based on the coupling of SLMs with a simplified optical setup. In order to display the complex information of the holograms we make use of two LCDs, one for the amplitude and one for the phase, in a cascade configuration. Usually the phase information is imaged into the plane where the amplitude information is placed by means of a $4 \mathrm{f}$ configuration and plane wave illumination. We propose a simplified setup that uses a single imaging lens to geometrically project the first LCD plane into the second one. In this case the illumination of the input image plays a significant role in the imaging process.

In Section 2 we present the method of displaying Fresnel holograms using two LCDs by separating the complex information into modulus and phase. The implementation of this method with real devices involves the adaptation of the complex function to the available modulation values of the
LCDs. This procedure is illustrated in Section 3 with some simulated results, which show Fresnel holograms that focus the information at different distances. We also compare the adjustment method with another procedure developed in [7]. In Section 4 the optical setup to apply this method is proposed and optical results are presented.

\section{Displaying full-complex Fresnel holograms on LCDs}

A hologram is a complex function that can be represented by a modulus and a phase. Therefore, in order to display such a function, we need a device able to modulate light in amplitude and phase, i.e., a spatial light modulator. The most common SLMs used in optical image processing are the liquid crystal displays. These devices modulate light depending on the applied voltage and the polarization state of the incident light. The behavior of an LCD can be represented by an operating curve, which gives information on the way this device modulates light depending on the gray level assigned to each pixel. The most common configurations used to display images on an LCD are [8]:

- High-contrast (HC): In this configuration the modulator contrast ratio should be as high as possible, although there may be a significant phase modulation.

- Amplitude-mostly (AM): In this case the LCD has to modulate light only in amplitude and the phase variation should be zero. In fact there is always a small phase modulation. With this configuration the contrast ratio is usually much lower than with the $\mathrm{HC}$ one.

- Phase-mostly (PM): Unlike the AM configuration, with this operating curve the light should be modulated only in phase but actually there is always a small variation in amplitude.

In an ideal case, we would need an LCD able to modulate simultaneously amplitude and phase in a wide range of values (a spiral-like operating curve, for example). This kind of configuration is almost unreachable with the available panels in the market. However, if we still want to use a single modulator to display full-complex values, it is 
possible to use codifying methods $[1,2]$ at the expense of a reduction in resolution.

As many LCDs can operate in AM and PM configurations the most suitable method for displaying full-complex information is to use a pair of panels, one for modulus and the other for phase. In this case we can avoid the limitations of the codifying methods when using a single modulator.

The use of two panels entails a significant problem: it is not possible to achieve the ideal case of having modulus and phase in the same plane, due to the thickness of the devices. Therefore we need an optical setup to have both set of data superimposed on a single plane by imaging one panel one-to-one (1:1) into the other, as we will see in Section 4.

As an example of complex information distribution to be displayed on the LCDs we use Fresnel holograms. We compute these digital holograms in order to recover an object at a certain distance from the plane where they are displayed. We start retropropagating the information of an image using the inverse Fresnel transform to a distance $-d$ as follows.

Let $f(x, y)$ be the original object to be codified in the hologram. Its Fresnel retropropagation to a distance $-d$ is

$$
\begin{aligned}
U(x, y,-d)= & \frac{\mathrm{e}^{-\mathrm{i} k d}}{-\mathrm{i} \lambda d} \int_{-\infty}^{\infty} f\left(x^{\prime}, y^{\prime}\right) \exp \left[-\frac{\mathrm{i} k}{2 d}\left(\left(x-x^{\prime}\right)^{2}\right.\right. \\
& \left.\left.+\left(y-y^{\prime}\right)^{2}\right)\right] \mathrm{d} x^{\prime} \mathrm{d} y^{\prime}
\end{aligned}
$$

where $k=2 \pi / \lambda$ is the wave number, and $(x, y)$ and $\left(x^{\prime}, y^{\prime}\right)$ are the transversal coordinates in the planes $z=-d$ and $z=0$, respectively.

The information to be displayed, $U(x, y,-d)$, is a complex function that can be represented as a modulus, $A(x, y,-d)$ and a phase, $\phi(x, y,-d)$

$$
U(x, y,-d)=A(x, y,-d) \mathrm{e}^{\mathrm{i} \phi(x, y,-d)} .
$$

When modulus and phase are displayed on the same plane and are illuminated by a plane wave, the original object $f(x, y)$ is reconstructed at a distance $d$ due to light propagation, as desired.

With this method it is possible to recover several objects at different planes simultaneously using a single digital hologram. We have to compute the Fresnel transform (Eq. (1)) for each object at its corresponding distance. Then, adding all the computed distributions we obtain a single complex function (modulus and phase) to be displayed on the panels. When illuminating this function, the different objects are recovered at their corresponding distances.

Gonçalves et al. [7] also use two LCD panels working in AM and PM configurations. But, instead of displaying modulus and phase separately, they consider an overall realizable complex transmittance as the product of the complex transmittances of the two LCDs. Then every value of a hologram is represented as a product of two of the available gray levels of each panel. When a modulator can operate in a phase-mostly mode that reaches $2 \pi$ it is possible to display full-complex information, otherwise there are values that cannot be represented. Nowadays, commercial LCDs are not usually designed to provide a full $2 \pi$ phase modulation because they are not thick enough [9], so its no possible to attain the whole complex plane.

\subsection{Fresnel holograms with EPSON VGA devices}

We want to display Fresnel holograms using the proposed method of displaying modulus and phase separately. We use a pair of LCDs removed from an EPSON VGA video projector EMP-3000. These panels offer a good performance in terms of pixelto-pixel addressing, which is a desired property to display holograms. However, these devices are limited by their resolution $(640 \times 480$ pixels and a pixel pitch of $42 \mu \mathrm{m}$ ) and their operating modes.

These LCDs were characterized by an interferometric method [10] in order to achieve three different configurations: HC, PM and AM. But we just obtained a suitable HC configuration (Fig. 1) and a PM curve that only introduces phase modulation between 0 and $\pi$ radians (Fig. 2). As with these curves it is not possible to fill the full-complex plane, it is necessary to adapt the data to fit the accessible modulation values.

We have chosen the HC curve as the most suitable mode to display the modulus because it offers a wide range of gray levels to represent positive real values. This configuration gives a contrast ratio of $124: 1$ and a maximum phase 


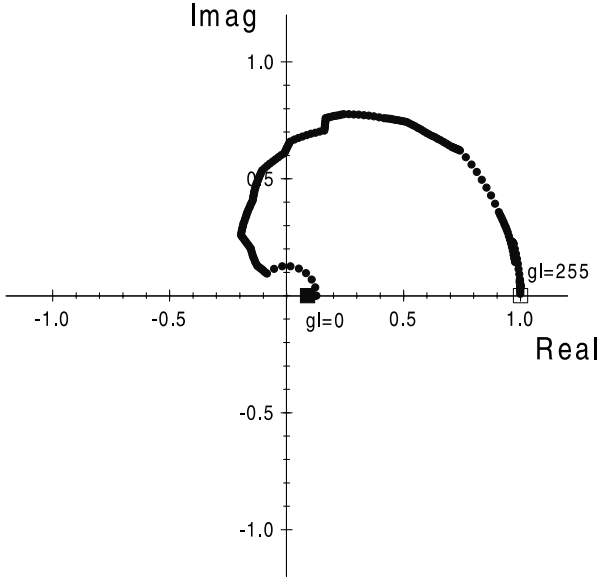

Fig. 1. High-contrast configuration.

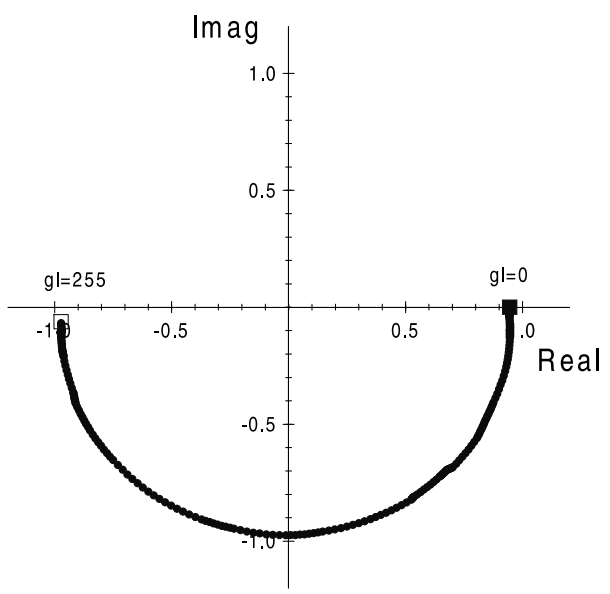

Fig. 2. $0-\pi$ Phase-mostly configuration.

modulation of $0.75 \pi$, where the contrast ratio is defined as the maximum intensity transmittance divided by the minimum intensity transmittance. We have fitted the values of the modulus to the available discrete levels of the curve using a criterion that finds the point that gives the nearest modulus without considering the phase.

The problem of the introduction of a significant amount of phase modulation when using this fitting method can be solved by compensating the values of the phase object. The compensation method consists in subtracting the phase values introduced by the $\mathrm{HC}$ configuration to the values of the computed phase.
Then, the compensated phase values are adapted to the PM configuration, which has a maximum phase modulation of $0.98 \pi$ and a contrast ratio of 1.1:1. In this case the adjustment is realized by a minimum Euclidean distance criterion (MED). Note that phase values between 0 and $\pi$ are binarized to 0 or $\pi$.

\section{Simulation results}

We have carried out some simulation experiments taking into account the modulation properties of the LCDs. We want to recover an image from a Fresnel hologram displayed on our VGA panels. The testing image is shown in Fig. 3. By using Eq. (1), we compute the complex values of the digital hologram at a distance of $-500 \mathrm{~mm}$. The modulus is fitted to the HC curve as described above, giving a residual phase that is used to obtain a compensated phase adapted to the PM curve.

Once we have the modulus and the compensated phase adapted to the configurations, we use them to compute the Fresnel diffraction at a distance of $500 \mathrm{~mm}$, where the initial object is recovered, as shown in Fig. 4. We have also simulated the reconstruction of objects at different distances, by using the building shadow and the letters in Fig. 3 as two independent objects to be recovered at distances of 500 and $600 \mathrm{~mm}$, respectively. The simulation results are shown in Figs. 5(a) and (b), where the object to be reconstructed at each distance appears focused while the other one is out of focus.

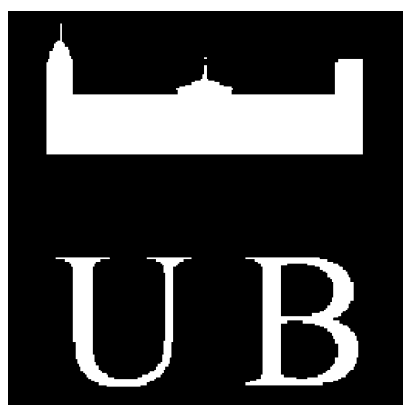

Fig. 3. Original object. 


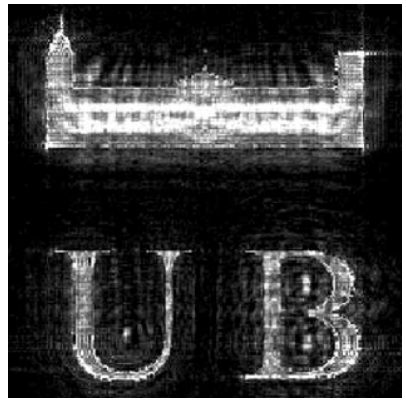

Fig. 4. Simulated reconstruction of the original object at $500 \mathrm{~mm}$.

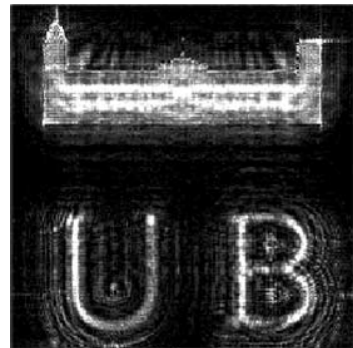

(a)

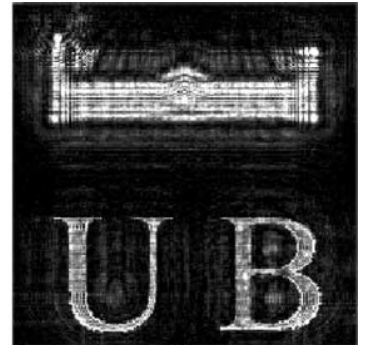

(b)
Fig. 5. Simulated recovered images: (a) at distance $500 \mathrm{~mm}$ (focusing the building), and (b) at $600 \mathrm{~mm}$ (focusing the letters).

The loss of quality of the reconstructions compared to the original object is mainly due to the limited modulation capabilities of the LCDs, specially the phase-mostly regime in which only a maximum phase of $\pi$ radians can be attained. Note that this is a consequence of the thickness of the VGA panels used, that are thinner than older CGA devices that provide $2 \pi$ radians of phase modulation $[7,10]$.

\subsection{Comparison of adjustment methods}

We also have carried out a study to analyze the differences between adjusting a complex function with the above explained method, which uses modulus and phase separately, or with the method that combines the accessible values of the two panels [7]. In order to apply the combined method we have calculated the complex values that can be displayed by combining the accessible configurations of our two panels. Fig. 6 shows the set of the

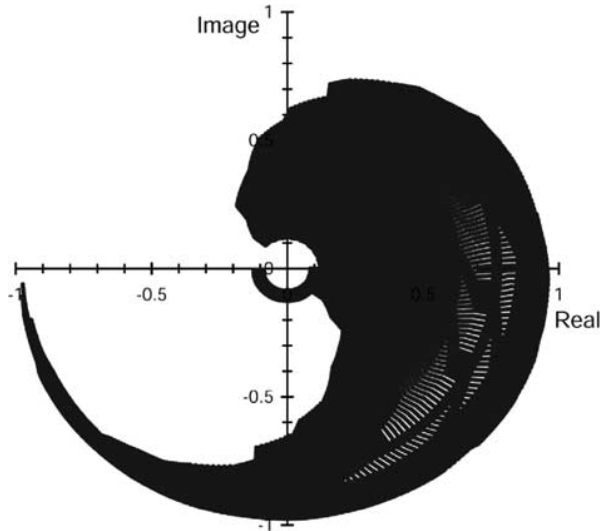

Fig. 6. Realizable complex plane coupling the achieved $\mathrm{HC}$ and PM curves.

accessible complex values that results from multiplying all the points of the PM configuration curve with the $\mathrm{HC}$ one. As the phase modulation provided by the PM mode is lower than $2 \pi$ radians, it is not possible to cover a complete circle in the complex plane. Therefore, there is a zone that cannot be directly represented, where it is necessary to use some kind of adjustment. We again use the minimum Euclidean distance criterion.

In order to evaluate the quality of the adjustment methods, we have calculated the root mean squared errors (RMS) of modulus and phase between the complex function to be displayed and the distribution adjusted to the devices, which is what is really displayed. The complex function that we use to calculate these RMS is the unity complex plane (see images of (a) modulus and (b) phase in Fig. 7). In Table 1 we can see the modulus

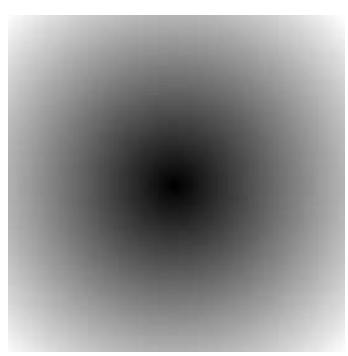

(a)

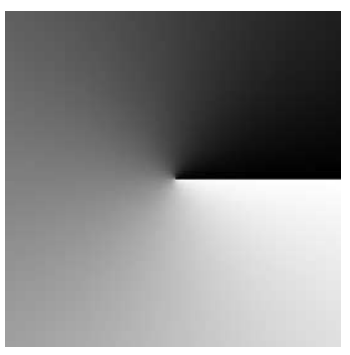

(b)
Fig. 7. Images of (a) the modulus and (b) the phase of the unity complex plane. 
Table 1

RMS error of modulus and phase for the separated and combined methods

\begin{tabular}{lll}
\hline RMS error & $\begin{array}{l}\text { Separated } \\
\text { method }(\%)\end{array}$ & $\begin{array}{l}\text { Combined } \\
\text { method }(\%)\end{array}$ \\
\hline Modulus & 3.5 & 16.4 \\
Phase & 9.7 & 2.9 \\
\hline
\end{tabular}

and phase RMS errors for the separated and the combined methods (see images of adjusted (a) modulus and (b) phase in Figs. 8 and 9, respec-

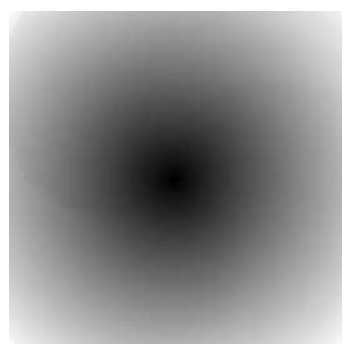

(a)

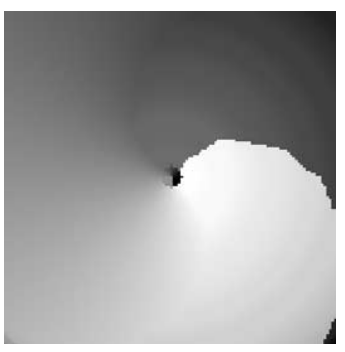

(b)
Fig. 8. Images of (a) the modulus and (b) the phase of the unity complex plane adjusted with the separated method.

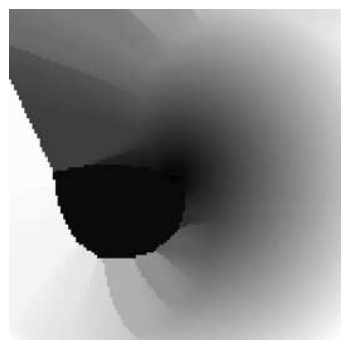

(a)

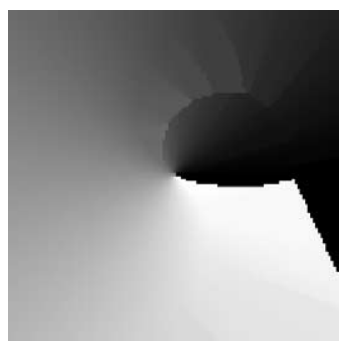

(b)
Fig. 9. Images of (a) the modulus and (b) the phase of the unity complex plane adjusted with the combined method. tively). The separated method works better when adjusting the modulus than when adjusting the phase. This behavior is opposite in the combined method, where the error in phase is lower than the error in modulus. When comparing both methods with each other, we find that the combined method is more robust when adjusting the phase while the separated method works better with the modulus. Thus, both methods give accurate results with irrelevant differences. However, the computing time for the separated method is much shorter than for the combined method, due to the different number of calculations required in the adjustment process. In the first case, the complex function to be displayed is separated in two, and then each one is adjusted to the accessible 256 values that correspond to the gray levels of each curve. In the second case, one single function is adjusted to $256^{2}$ values. For example, in a Pentium III computer at $800 \mathrm{MHz}$, a complex function of $512 \times 512$ pixels is adjusted in about $20 \mathrm{~s}$ and over $1 \mathrm{~h}$ using the separated or the combined method, respectively.

\section{Optical setup and experimental results}

We have designed an optical setup to implement our method for displaying Fresnel holograms with the real devices (Fig. 10). In order to have modulus and phase information placed in the same plane, the first LCD is imaged 1:1 onto the second one using a collimating lens with a focal length of $f$. This condition requires the panels to be situated symmetrically at distances $2 f$ from the lens. In fact there is a negative magnification that can be easily overcome rotating one of the images or one modulator $180^{\circ}$.

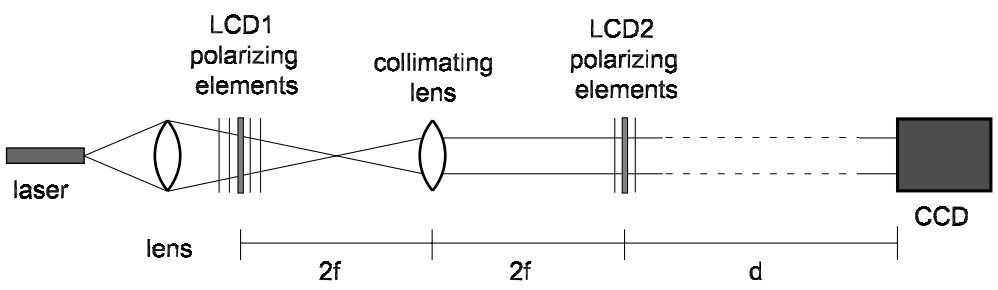

Fig. 10. Optical setup. 
The main drawback of this setup is the alignment of the displays to obtain the correct product of modulus and phase. This required pixel-to-pixel superimposition is achieved by controlling it with a microscope and a micropositioning system.

We propose this $2 \mathrm{f}$-cascade architecture because by using the appropriate illumination the undesirable phase terms that appear in the imaging process can be cancelled. This configuration is also simpler than the $4 \mathrm{f}$ configuration proposed by other authors $[4,5]$ by using less lenses.

When an object $s(x, y)$ is imaged through a lens the complex distribution that is formed in the image plane is the following [11]:

$t(x, y) \propto \exp \frac{\mathrm{i} k}{2 f}\left(x^{2}+y^{2}\right) s(-x,-y)$.

Considering the illumination of the object, which in our case is a converging wave, the input function $s(x, y)$ must be replaced by $s(x, y) \exp -(\mathrm{i} k /$ $2 f)\left(x^{2}+y^{2}\right)$ in Eq. (3). Therefore in the image plane we have

$t(x, y) \propto s(-x,-y)$.

Thus, illuminating the object with the suitable converging wave we cancel the spherical phase factor that appeared in Eq. (3). Then it is possible to have the phase and modulus information of the complex Fresnel hologram to recover superimposed on the same plane without other terms.

The described optical setup has been used to reconstruct the object shown in Fig. 3 from the computed Fresnel hologram, using two lenses with a focal length of $135 \mathrm{~mm}$. The compensated phase is displayed on the first LCD while the modulus is on the second modulator. The first LCD is placed between two linear polarizers and two quarter wave plates to achieve elliptical polarization suitable to obtain the PM operating curve. The second one only needs two linear polarizers for the $\mathrm{HC}$ configuration. A CCD camera is placed at a distance of $500 \mathrm{~mm}$ behind the second panel, which is the distance used to compute the Fresnel hologram. This camera captures the reconstruction of the object as shown in Fig. 11. We have also computed a hologram to recover the building shadow in Fig. 3 at a distance of $500 \mathrm{~mm}$ and the two letters in the same figure at a distance of

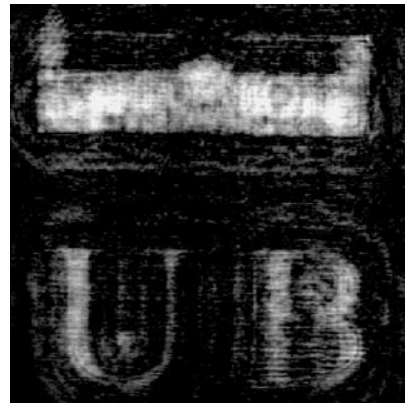

Fig. 11. Experimental reconstruction of the original object at $500 \mathrm{~mm}$.

$600 \mathrm{~mm}$. The respective captured planes are shown in Figs. 12(a) and (b). In these two figures we can see that the corresponding object is focused, while the other appears out of focus. We appreciate that the optical results agree with the simulated ones (Figs. 4 and 5(a) and (b)). The loss of quality is due to experimental difficulties such as non-uniform illumination, noise introduced by the polarizing elements, lack of parallelism between the panels and misalignments.

Amako et al. [6] use a different coupled setup to record Fourier holograms and obtain optical reconstructions with two LCDs specially designed for amplitude and phase modulation. Their optical setup is longer than the above proposed because it uses an additional lens to perform the Fourier transform of the information displayed on the panels. In our case the distance $d$ where the Fresnel holograms are recovered can be smaller and it is easier to tune. In [7] Fresnel holograms are reconstructed at a distance of $3 \mathrm{~m}$ by coupling two

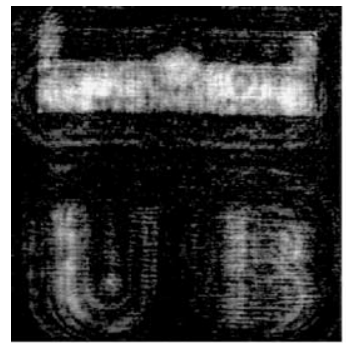

(a)

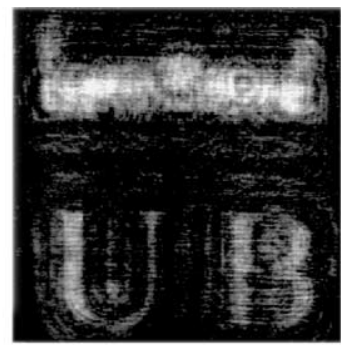

(b)
Fig. 12. Experimental recovered images: (a) at distance $500 \mathrm{~mm}$ (focusing the building), and (b) at $600 \mathrm{~mm}$ (focusing the letters). 
LCDs with another cascaded setup that makes use of three lenses instead of two, as in our case.

\section{Conclusion}

We have implemented a method for displaying complex Fresnel holograms by using two LCDs in a cascade configuration. The optical setup is based on the projection of the phase plane into the amplitude plane by means of an imaging lens. The method to adapt the complex information to real devices starts by adjusting the amplitude to a $\mathrm{HC}$ operating curve. The remaining phase introduced when adjusting the amplitude to a $\mathrm{HC}$ curve is subtracted to the true phase of the object. This compensated phase is then adapted to a PM curve by using a MED criterion. We have applied this procedure to record multiple objects at different distances in a single hologram. The simulation performed and the optical reconstructions show a reasonable agreement taking into account the limitations of the devices used, which only provide a maximum of $\pi$ phase modulation.

We have compared our adjustment method where modulus and phase are considered separately with a different procedure that combines the accessible values of the two panels. The quality of both adjustments is similar but the computation time is much shorter in our case. We have proposed a simplified setup that uses a single imaging lens to geometrically project the first LCD plane into the second one, and a converging illuminating wave. With such setup we reduce the number of required lenses when using two LCDs in a cascade architecture.

\section{Acknowledgements}

This paper has been partially funded by the CICYT (Comisión Interministerial de Ciencia Y Tecnologia) through project DPI 2001-3365.

\section{References}

[1] G. Tricoles, Appl. Opt. 26 (1987) 4351.

[2] P. Birch, R. Young, C. Chatwin, M. Farsari, D. Budgett, J. Richardson, Opt. Commun. 175 (2000) 347.

[3] C. Stolz, L. Bigué, P. Ambs, Appl. Opt. 40 (2001) 6415.

[4] R.D. Juday, J.M. Florence, Proc. SPIE 1558 (1991) 499.

[5] D.A. Gregory, J.C. Kirsch, E.C. Tam, Appl. Opt. 31 (1992) 163.

[6] J. Amako, H. Miura, T. Sonehara, Appl. Opt. 32 (1993) 4323.

[7] L. Gonçalves Neto, D. Roberge, Y. Sheng, Appl. Opt. 35 (1996) 4567.

[8] C. Soutar, S.E. Monroe Jr., Proc. SPIE 2240 (1994) 280.

[9] I. Labastida, A. Carnicer, E. Martin-Badosa, S. Vallmitjana, I. Juvells, Appl. Opt. 39 (2000) 766.

[10] E. Martín-Badosa, A. Carnicer, I. Juvells, S. Vallmitjana, Meas. Sci. Technol. 8 (1997) 764.

[11] A. VanderLugt, Optical Signal Processing, John Wiley and Sons, New York, 1992. 